\title{
ON SELECTING THE BEST OF TWO NORMAL POPULATIONS USING A LOSS FUNCTION
}

\author{
Paul van der Laan \\ Constance van Eeden \\ Eindhoven University of Technology \\ University of British Columbia \\ P.O. Box 513 \\ Université du Québec à Montréal \\ 5600 MB Eindhoven \\ Moerland 19 \\ The Netherlands \\ 1151 BH Broek in Waterland \\ The Netherlands
}

Key words: Subset selection; selection; probability of correct selection; loss function; $\varepsilon$-best population; generalized selection goal.

\begin{abstract}
In selection problems the usual loss function is the 0 - 1 one, i.e. the selection goal is to bound, from below, probabilities of making "correct" selections. In the present paper a selection goal based on a general loss function is presented. The populations have unknown location parameters and "good" populations are the ones with large values of this parameter. The case of two populations is considered in detail. For this case the selection rule is given and its performance is investigated for normal populations.
\end{abstract}

\section{INTRODUCTION}

Statistical selection methods are concerned with the problem of selecting, out of a set of $k$ populations $\pi_{1}, \cdots, \pi_{k}$, a non-empty subset containing the "best" population. This selection is made on the basis of $k$ independent samples, one from each 
population. Here, the "best" population is, in most cases, defined as the one with the largest (or smallest) value of an unknown parameter, e.g. the mean, or the variance. An agricultural experimenter, e.g., might be interested in the variety with the highest yield. In the case where the parameter is the variance one might want to find the population with the smallest value of this parameter.

There are two basic approaches to subset selection. One approach, the indifference zone approach, was suggested by Bechhofer (1954). The other approach, subset selection, was presented by Gupta $(1956,1965)$. Hsu's (1984) confidence-bound approach encompasses Bechhofers's as well as Gupta's approach. The indifference zone approach has as its goal the selection of the best population. The sample sizes are chosen in such a way that, for some given $P^{*} \in\left(\frac{1}{k}, 1\right)$, the probability of a correct selection is at least $P^{*}$ whenever the best population is at least $\delta^{*}\left(\delta^{*}>0\right.$, given $)$ away from the second best. The subset selection approach has as its goal the selection of a non-empty subset, as small as possible, containing the best population with a given probability. And this probability requirement has to be met for all possible parameter values.

Practical objections to selection procedures are of the type: "large sample sizes needed" for the indifference zone approach and "large subsets" for the subset selection approach. For strong requirements (large $P^{*}$ and/or small $\delta^{*}$ ), when using the indifference zone approach, one has to pay with large sample sizes. Using the subset selection approach one pays, for fixed sample sizes, with a large size of the selected subset. These large subsets are mainly due to the fact that the probability requirement has to be met also for the least favourable configuration (LFC) of the parameters. For the location model the LFC is, in many cases, the configuration consisting of equal parameter values for the $k$ populations.

Generalizations of the above approaches where, instead of looking for the best population, the goal is to find the best $t(2 \leq t<k)$ populations have also been considered (see e.g. Gupta and Panchapakesan (1979) for these and other modifications).

The performance of selection procedures can be improved by either increasing the sample sizes or by weakening the probability requirements. An investigator might 
not be able or willing to do either. However, he might be able to specify an $\varepsilon>0$ such that, from a practical point of view, he is content when his subset contains an $\varepsilon$-best population. Here, an $\varepsilon$-best population is any population with a parameter value within $\varepsilon$ of the value of the largest parameter. This concept of $\varepsilon$-best started, in fact, with the notion of $\Delta$-correct ranking introduced by Fabian (1962) and Lehmann (1963). There are several papers dealing with the problem of selecting all good populations, which is the same as that of all $\varepsilon$-best populations, with a predescribed confidence or to select a subset which excludes all inferior populations with a minimal guaranteed probability $P^{*}$, where an inferior population is defined by a parameter value not within $\varepsilon^{*}(>\varepsilon)$ of the largest parameter value (Desu, 1970). Similar ideas have been developed in the context of the $t$-th best population (see for instance Panchapakesan and Santner (1977)). For the location problem, van der Laan (1992) studied the use of an $\varepsilon$-best population in subset selection with as goal the selection of a non-empty subset which includes at least one $\varepsilon$-best population with a minimal guaranteed probability $P^{*}\left(\frac{1}{k}<P^{*}<1\right)$. Gill and Sharma (1993) consider the location problem for exponential populations. They propose a procedure which omits an $\varepsilon$-best population or includes an inferior one with probability at most $1-P^{*}$. Desu and Bristol (1985) propose a procedure where one of the possible decisions is to not make a selection and declare the populations to be "equivalent". For an overview of the literature on this subject we refer to the survey paper by Gupta and Panchapakesan (1985).

In the present paper a further generalization is considered. It consists of replacing the zero-one loss function by a continuous one. There are several papers dealing with the problem of selection using a decision-theoretic approach based on loss functions. Some of them are dealing with Bayes procedures using loss functions of various forms. To mention a few: Studden (1967), Deely and Gupta (1968), Bickel and Yahav (1977) and Gupta and Kim (1981). There also are papers which consider a loss for every good population which is not selected and for every bad population which is selected. For references, for such cases, we refer to the survey paper by Gupta and Panchapakesan (1985) and to Lam and Yu (1994). Lam and Yu (1994) consider two 
types of loss functions, where one measures the loss of including bad populations and the other one measures the loss of the non-selection of good populations. We consider a location problem and our loss function takes into account the difference, in parameter value, between the best population in the selected subset and the best of all populations minus $\varepsilon$ ( $\varepsilon \geq 0$, given). Although the case of two normal populations is considered in this paper, a general description of our generalization for the case of $k(k \geq 2)$ populations is given in Section 2. In Section 3 the special case of two normal populations is studied in detail. Section 4 contains a discussion and a comparison, for the case of two normal populations, of our results with those of Gupta and van der Laan. Section 5 contains some lemmas used in the proofs of our results. Section 6 contains tables needed when our results are applied in the two-sample normal case with a quadratic loss function.

\section{A GENERALIZED SUBSET SELECTION GOAL}

Let, for $i=1, \cdots, k, X_{i 1}, \cdots, X_{i n_{i}}$ be a sample from $\pi_{i}$ and suppose these $k$ samples are independent. It is assumed that $X_{i 1}$ has distribution function $F\left(x-\theta_{i}\right)$, where $\theta_{i} \in \Theta \subset \mathbb{R}$ is unknown, $i=1, \cdots, k$, and $F(x)$ is a known function of $x$. The ordered parameters are denoted by $\theta_{[1]} \leq \cdots \leq \theta_{[k]}$ and the population associated with $\theta_{[i]}$ is denoted by $\pi_{(i)}, i=1, \cdots, k$. The best population is $\pi_{(k)}$. Finally, the selected subset of indices from $K=\{1, \cdots, k\}$ is denoted by $d$.

The generalized loss function considered in this paper is of the form

$$
L(\theta, d, \varepsilon)=h\left(\theta_{[k]}-\varepsilon-\max _{i \in d} \theta_{i}\right) I\left(\max _{i \in d} \theta_{i}<\theta_{[k]}-\varepsilon\right),
$$

with $\theta=\left(\theta_{1}, \cdots, \theta_{k}\right), \varepsilon \geq 0, h$ a non-decreasing function defined on $\mathbb{I R}^{+}$and $\mathrm{I}(\mathrm{A})$ the indicator function of the set $\mathrm{A}$. The subset selection approach studied by van der Laan (1992) corresponds to $h(x)=1$ for all $x \in \mathbb{R}^{+}$. The advantage of our approach is that it does not just take into account whether or not an $\varepsilon$-best population is in the subset, but the loss function measures how bad the best one in the subset is if 
no $\varepsilon$-best population is there. Note that we consider only losses due to the absence of good populations in the selected subset and no losses due to the presence of bad populations. Our procedure attains a goal similar to "not too often too many bad ones in the selected subset" by bounding the risk function $R$ and/or the expected subset size. For $k=2$, where there is at most one bad one in the subset, choosing a rule such that $R \leq R_{0}$ when $\theta_{[2]}-\theta_{[1]}$ is large gives the procedure a risk $\leq R_{0}$ when the bad population is really bad. Further, putting an upperbound on the expected subset size for large values of $\theta_{[2]}-\theta_{[1]}$ gives another protection against the presence of a bad one too often. In fact, when $k=2$, an upperbound on the expected subset size is equivalent to an upperbound on the probability that the subset size equals 2. The question of when the rule can be chosen to satisfy these two conditions simultaneously is discussed below. Also, in Section 4 some numerical results will be presented comparing our procedure with Gupta's $(1956,1965)$ and with the one studied by van der Laan (1992). In particular, the probability that our subset contains the best, the worst and an $\varepsilon$-best will be computed for several examples. In the next section the case of two normal populations, with equal variances and $h(x)=x^{p}, p>1, x \in \mathbb{R}^{+}$, is studied in detail.

\section{THE CASE OF TWO NORMAL POPULATIONS}

Let $k=2, n_{1}=n_{2}=n$ and let $X_{i 1}$ be $N\left(\theta_{i}, 1\right), i=1,2$. Taking, for some $p>1, h(x)=x^{p}, x \in \mathbb{R}^{+}$, the loss function becomes $L(\theta, d, \varepsilon)=\left(\theta_{[2]}-\varepsilon-\right.$ $\left.\theta_{[1]}\right)^{p} I\left(\max _{i \in d} \theta_{i}<\theta_{[2]}-\varepsilon\right)$. The decision rules, $\delta_{c}$, considered in this section are of the form

$$
\delta_{c}(x)\left\{\begin{array}{lll}
d_{1}=\{1\} & \text { if } & \bar{x}_{1}-\bar{x}_{2}>c \\
d_{2}=\{2\} & \text { if } & \bar{x}_{1}-\bar{x}_{2}<-c \\
d_{3}=\{1,2\} & \text { if } & \left|\bar{x}_{1}-\bar{x}_{2}\right| \leq c
\end{array}\right.
$$

where $\bar{x}_{i}=\frac{1}{n} \sum_{j=1}^{n} x_{i j}, i=1,2$, are the observed means and where $c \geq 0$ is a constant. 
These rules are of the same form as the ones used by Bechhofer (1954) and by Gupta $(1956,1965)$. Also the rule studied in van der Laan (1992) is of the same form. The difference between the various approaches is in the criteria used for the choice of $c$. Bechhofer takes $c=0$ and chooses $n$ in such a way that $P_{\theta}\left(\theta_{d}=\theta_{[2]}\right) \geq$ $P^{*}$ for all $\theta$ with $\theta_{[2]}-\theta_{[1]} \geq \delta^{*}$, for some given pair $\left(P^{*} \in\left(\frac{1}{k}, 1\right), \delta^{*}>0\right)$, and where $\theta_{d}$ is the $\theta$ value associated with the largest observed mean. Gupta chooses $c$ such that, for some given $\alpha \in\left(\frac{1}{k}, 1\right), P_{\theta}\left(\theta_{j}=\theta_{[2]}\right.$ for some $\left.j \in d\right) \geq 1-\alpha$ for all $\theta$, with $d$ the smallest subset of $K$ for which this goal can be achieved. If $\theta_{1}=\theta_{2}$ then Gupta takes $\theta_{[2]}$ to be a flagged value. The selection rule studied by van der Laan uses, for fixed $\varepsilon>0$ and $\alpha \in\left(\frac{1}{k}, 1\right)$, the constant $c$ such that $P_{\theta}\left(\max _{i \in d} \theta_{i} \geq \theta_{[2]}-\varepsilon\right) \geq$ $1-\alpha$ for all $\theta$, where, as for the Gupta approach, $d$ is the smallest such subset.

In this section we consider an upper bound on $\mathcal{E}_{\theta} L\left(\theta, \delta_{c}, \varepsilon\right)$ and/or on $\mathcal{E}_{\theta} S_{c}$, where $S_{c}$ is the size of the selected subset obtained when using the decision rule $\delta_{c}$. To simplify the notation, the bars on $\bar{x}_{i}, i=1,2$, will be left off.

We first obtain, in the next two theorems, expressions for the risk function and for the expected subset size as functions of $\theta, c, \varepsilon$ and $n$.

Theorem 3.1. The risk function of the rule $\delta_{c}$ is given by

$$
R\left(\mu, \delta_{c}, \varepsilon\right)=\mathcal{E}_{\theta} L\left(\theta, \delta_{c}, \varepsilon\right)=(\mu-\varepsilon)^{p} \Phi\left(\frac{-c-\mu}{\sqrt{2}} \sqrt{n}\right) I(\mu>\varepsilon)
$$

where $\mu=\left|\theta_{1}-\theta_{2}\right|$ and $\Phi(\cdot)$ is the standard normal distribution function.

Proof. First note that

$$
\begin{aligned}
& L\left(\theta, d_{1}, \varepsilon\right)=\left(\theta_{2}-\varepsilon-\theta_{1}\right)^{p} I\left(\theta_{1}<\theta_{2}-\varepsilon\right) \\
& L\left(\theta, d_{2}, \varepsilon\right)=\left(\theta_{1}-\varepsilon-\theta_{2}\right)^{p} I\left(\theta_{2}<\theta_{1}-\varepsilon\right) \\
& L\left(\theta, d_{12}, \varepsilon\right)=0 \text { for all } \theta .
\end{aligned}
$$

Thus 


$$
R\left(\mu, \delta_{c}, \varepsilon\right)=\left(\left|\theta_{2}-\theta_{1}\right|-\varepsilon\right)^{p} P_{\theta}\left(X_{1}-X_{2}>c\right) I\left(\left|\theta_{2}-\theta_{1}\right|>\varepsilon\right),
$$

obtained by using the fact that $P_{\theta}\left(X_{1}-X_{2}>c \mid \theta_{1}<\theta_{2}-\varepsilon\right)=P_{\theta}\left(X_{1}-X_{2}<-c \mid \theta_{2}<\right.$ $\left.\theta_{1}-\varepsilon\right)$. The result then follows from the fact that $X_{1}-X_{2}$ has a $N\left(\theta_{1}-\theta_{2}, \frac{2}{n}\right)$ distribution.

Theorem 3.2. The expected subset size is given by

$$
\mathcal{E}_{\theta} S_{c}=\Phi\left(\frac{c-\mu}{\sqrt{2}} \sqrt{n}\right)+\Phi\left(\frac{c+\mu}{\sqrt{2}} \sqrt{n}\right)
$$

Proof. The result follows immediately from

$$
\begin{aligned}
\mathcal{E}_{\theta} S_{c} & =P_{\theta}\left(\left|X_{1}-X_{2}\right|>c\right)+2 P_{\theta}\left(\left|X_{1}-X_{2}\right| \leq-c\right) \\
& =\Phi\left(\frac{c-\mu}{\sqrt{2}} \sqrt{n}\right)+\Phi\left(\frac{c+\mu}{\sqrt{2}} \sqrt{n}\right) .
\end{aligned}
$$

Some properties of $R\left(\mu, \delta_{c}, \varepsilon\right)$ and $\mathcal{E}_{\theta} S_{c}$, as functions of $\mu, c$ and $\varepsilon$, are stated and proved in Section 5. These properties are used below to study the behaviour of $\delta_{c}$ when, for a fixed $\varepsilon$, an upper bound, $R_{0}$, is put on $R\left(\mu, \delta_{c}, \varepsilon\right)$. We will also, for a fixed $R_{0}$, look at the influence of $\varepsilon$ on $\delta_{c}$, and study $\mathcal{E}_{\theta} S_{c}$ as a function of $R_{0}$.

First note that, by Lemma 5.2 , part iii), $\max _{\mu \geq 0} R\left(\mu, \delta_{c}, \varepsilon\right)$ is, for each $\varepsilon$, continuous and strictly decreasing in $c$ and converges to 0 as $c \rightarrow \infty$. So, for any $\varepsilon$ and any $R_{0} \in\left(0, \max _{\mu \geq 0} R\left(\mu, \delta_{0}, \varepsilon\right)\right)$, there exists a $c\left(\varepsilon, R_{0}\right)$ such that $\max _{\mu \geq 0} R\left(\mu, \delta_{c}, \varepsilon\right)<,=,>$ $R_{0}$ according as $c>,=,<c\left(\varepsilon, R_{0}\right)$. Further (see Theorem 3.2 and Lemma 5.3, part ii)), $\mathcal{E}_{\theta} S_{c}$ is, for each fixed $\mu$, a strictly increasing function in $c$. So, if one requires of the procedure that $\max _{\mu \geq 0} R\left(\mu, \delta_{c}, \varepsilon\right)<R_{0}$ with $\max _{\mu} \mathcal{E}_{\theta} S_{c}$ as small as possible, then $\delta_{c\left(\varepsilon, R_{0}\right)}$ is the unique rule satisfying this requirement.

Further, by Lemma 5.2, part iv), $\max _{\mu \geq 0} R\left(\mu, \delta_{c}, \varepsilon\right)$ is decreasing in $\varepsilon$. So $c\left(\varepsilon, R_{0}\right)$ is decreasing in $\varepsilon$ (in fact, it is easy to see that $c\left(\varepsilon, R_{0}\right)=c\left(0, R_{0}\right)-\varepsilon$ ) and this implies that $\max _{\mu \geq 0} \mathcal{E}_{\theta} S_{c\left(\varepsilon, R_{0}\right)}$ is decreasing in $\varepsilon$.

Another way to decrease the expected subset size is to require that the bound $R_{0}$ 
holds only for some values of $\mu$. By Lemma 5.1, parts iii) and iv), there then is, when $R_{0} \in\left(0, \max _{\mu \geq 0} R\left(\mu, \delta_{c}, \varepsilon\right)\right)$, an interval $I\left(R_{0}, c, \varepsilon\right)=\left(\mathrm{a}\left(R_{0}, c, \varepsilon\right), \mathrm{b}\left(R_{0}, c, \varepsilon\right)\right)$ such that $R\left(\mu, \delta_{c}, \varepsilon\right)>R_{0}$ for $\mu \in I\left(R_{0}, c, \varepsilon\right)$, and $\leq R_{0}$, elsewhere. By the same reasoning as above one sees that the smallest $\max _{\mu \geq 0} \mathcal{E}_{\theta} S_{c}$ for which $\max _{a \leq \mu \leq b} R\left(\mu, \delta_{c}, \varepsilon\right) \leq R_{0}$ can be achieved for given $(a, b)$ with $\varepsilon \leq a<b$, is decreasing in $R_{0}$ and decreasing in $\varepsilon$.

Finally, one could require that $\max _{\mu \geq 0} \mathcal{E}_{\theta} S_{c} \leq 1+\eta_{0}$ for some given $\eta_{0} \in(0,1)$. This would lead to an upper bound on $c$ and thus to a lower bound, $R\left(\eta_{0}\right)$, on $\max _{\mu \geq 0} R\left(\mu, \delta_{c}, \varepsilon\right)$. The only way to then achieve $\max _{\mu \geq 0} R\left(\mu, \delta_{c}, \varepsilon\right)<R_{0}$ in case $R\left(\eta_{0}\right)>$ $R_{0}$ is to increase $\varepsilon$. Note that $\mathcal{E}_{\theta} S_{c}=1+P_{\theta}\left(S_{c}=2\right)$, so $\max _{\mu \geq 0} \mathcal{E}_{\theta} S_{c} \leq 1+\eta_{0}$ is equivalent to $\max _{\mu \geq 0} P_{\theta}\left(S_{c}=2\right) \leq \eta_{0}$.

In order to apply in practice the above subset selection techniques, the values of $R\left(\mu, \delta_{c}, \varepsilon\right), \mu_{m}(c, \varepsilon)$ (defined in Lemma 5.1), $\max _{\mu \geq 0} R\left(\mu, \delta_{c}, \varepsilon\right), \mathcal{E}_{\theta} S_{c}$ and of $a\left(R_{0}, c, \varepsilon\right)$ and $b\left(R_{0}, c, \varepsilon\right)$ are needed. The values of $R\left(\mu, \delta_{c}, \varepsilon\right)$ and $\mathcal{E}_{\theta} S_{c}$ can easily be obtained from their formulas given in Theorem 3.1 and Theorem 3.2. Tables of $\mu_{m}(c, \varepsilon)$ and of $a\left(R_{0}, c, \varepsilon\right)$ and $b\left(R_{0}, c, \varepsilon\right)$ have been computed for the case when $p=2, n=1, \varepsilon=0$. These tables can be found in Section 6 . Values of these quantities for the case where $n>1$ or $\varepsilon>0$ can be obtained by using the fact that (with an obvious change in notation), for all $n \geq 1$ and $\varepsilon \geq 0$,

$$
\begin{array}{ll}
\mu_{m, n}(c, \varepsilon)=\mu_{m, n}(c, 0)+\varepsilon & \mu_{m, n}(c, \varepsilon)=n^{-\frac{1}{2}} \mu_{m, 1}\left(c^{*}, \varepsilon^{*}\right) \\
a_{n}\left(R_{0}, \delta_{c}, \varepsilon\right)=a_{n}\left(R_{0}, \delta_{c+\varepsilon}, 0\right)+\varepsilon & a_{n}\left(R_{0}, c, \varepsilon\right)=n^{-\frac{1}{2}} a_{1}\left(n^{\frac{p}{2}} R_{0}, c^{*}, \varepsilon^{*}\right) \\
b_{n}\left(R_{0}, \delta_{c}, \varepsilon\right)=b_{n}\left(R_{0}, \delta_{c+\varepsilon}, 0\right)+\varepsilon & b_{n}\left(R_{0}, c, \varepsilon\right)=n^{-\frac{1}{2}} b_{1}\left(n^{\frac{p}{2}} R_{0}, c^{*}, \varepsilon^{*}\right),
\end{array}
$$

where $c^{*}=c \sqrt{n}$ and $\varepsilon^{*}=\varepsilon \sqrt{n}$. Finally, $\max _{\mu \geq 0} R\left(\mu, \delta_{c}, \varepsilon\right)=R\left(\mu_{m}(c, \varepsilon), \delta_{c}, \varepsilon\right)$.

\section{DISCUSSION OF THE PROCEDURE AND COMPARISON WITH GUPTA'S}

The advantages and disadvantages of the loss function approach, Gupta's (1956, 
1965) subset selection approach and the $\varepsilon$-best approach studied by van der Laan (1992) will now be discussed by means of some numerical examples.

A disadvantage of the loss function approach is that a loss function has to be specified. And, when using a loss function, new tables, as the ones presented in this paper, are necessary. These tables are not easy to make. An advantage of the loss function approach is that it does not just take into account whether or not an $\varepsilon$-best population is an element of the subset, but how bad the best population in the selected subset is. The above loss function approach also has a greater flexibility, in that two aspects of the decision procedure can be controlled: the expected loss or risk $R\left(\mu, \delta_{c}, \varepsilon\right)$ and the expected subset size $\mathcal{E}_{\theta} S_{c}$. Upper bounds can be fixed for both. Expected loss and expected subset size are not comparable or exchangeable characteristics and we are therefore dealing with these characteristics separately in the presented selection procedure.

The difference between the loss function approach and Gupta's will now be illustrated by means of some numerical examples. In these examples it is assumed, without loss of generality, that $\theta_{1}<\theta_{2}$. The selection constant $c$ is chosen in such a way that $\min _{\mu \geq 0} P_{\theta}\left(\pi_{2}\right.$ in subset $)=.65$ for the case $n=5$ as well as for the case $n=10$. The first table below contains $\min _{\mu \geq 0} P_{\theta}$ (an $\varepsilon$-best in subset) for $\varepsilon=0$ and for $\varepsilon=.2$. This table also contains $\max _{\mu>\mu_{1}} P_{\theta}\left(\pi_{1}\right.$ in subset $)$ for $\mu_{1}=1$ and for $\mu_{1}=1.5$. The second table contains values of the risk function and of the expected subset size for various values of $\mu$. 
TABLE 4.1. $\operatorname{Min}_{\mu \geq 0} P_{\theta}$ (an $\varepsilon$-best is in the selected subset $C$ ) and $\max _{\mu \geq \mu_{1}} P_{\theta}\left(\pi_{1}\right.$ is in the selected subset $C$ ) for some values of $\varepsilon, \mu_{1}$ and the common sample size $n$

\begin{tabular}{|r|l|l|l|l|}
\hline \multirow{2}{*}{$n$} & \multicolumn{2}{|c|}{$\min _{\mu \geq 0} P_{\theta}($ an $\varepsilon$-best $\in C)$} & \multicolumn{2}{|c|}{$\max _{\mu \geq \mu_{1}} P_{\theta}\left(\pi_{1} \in C\right)$} \\
\cline { 2 - 5 } & $\varepsilon=0$ & $\varepsilon=.2$ & $\mu_{1}=1$ & $\mu_{1}=1.5$ \\
\hline 5 & .65 & .76 & .12 & .024 \\
10 & .65 & .80 & .032 & .0015 \\
\hline
\end{tabular}

TABLE 4.2. Some values of the risk function $R\left(\mu, \delta_{c}, \varepsilon\right)$ for $\varepsilon=.2$ and the expected subset size $\mathcal{E}_{\theta} S_{c}$

\begin{tabular}{|r|l|l|l|l|l|l|}
\hline \multirow{2}{*}{$n$} & \multicolumn{3}{|c|}{$R\left(\mu, \delta_{c}, \varepsilon\right)$} & \multicolumn{3}{c|}{$\max _{\mu \geq \mu_{1}} \mathcal{E}_{\theta} S_{c}$} \\
\cline { 2 - 7 } & $\max _{\mu \geq .2}$ & $\mu=1$ & $\mu=1.5$ & $\mu_{1}=0$ & $\mu_{1}=1$ & $\mu_{1}=1.5$ \\
\hline 5 & .018 & .016 & .0049 & 1.30 & 1.11 & 1.08 \\
10 & .0067 & .0027 & .00034 & 1.30 & 1.08 & 1.06 \\
\hline
\end{tabular}

The results in the Tables 4.1 and 4.2 are found by using the Theorems 3.1 and 3.2 , Table 6.1 and the relations given at the end of Section 3 .

For the cases considered in the two tables one sees that the probability of selecting an $\varepsilon$-best population can be substantially larger than the probability of selecting the best one. The difference is increasing with $n$. The probability of selecting the worst population is small and decreases rapidly with $n$. From Table 4.2 it is seen that $R$ decreases rapidly with increasing $\mu$ and also with increasing $n$. The expected subset 
size is close to one, decreases with $n$ and also with $\mu$.

\section{SOME LEMMAS}

In this section some properties of the risk function $R\left(\mu, \delta_{c}, \varepsilon\right)$ and the expected subset size $\mathcal{E}_{\theta} S_{c}$ as functions of $\mu, c$ and $\varepsilon$ are stated and proved.

Lem ma 5.1. The risk function $R\left(\mu, \delta_{c}, \varepsilon\right)$ satisfies, for each $\varepsilon$,

i) $R\left(\mu, \delta_{c}, \varepsilon\right)=0$ for all $c \geq 0$ when $\mu \leq \varepsilon$,

ii) for each $\mu>\varepsilon, R\left(\mu, \delta_{c}, \varepsilon\right)$ is strictly decreasing in $c$,

iii) for each $c, R\left(\mu, \delta_{c}, \varepsilon\right)$ is strictly increasing in $\mu$ on $\left(\varepsilon, \mu_{m}(c, \varepsilon)\right)$, strictly decreasing in $\mu$ on $\left(\mu_{m}(c, \varepsilon), \infty\right)$,

iv) for each $c, R\left(\mu, \delta_{c}, \varepsilon\right)$ converges to 0 as $\mu \rightarrow \infty$.

Proof. The proofs of i), ii) and iv) are straightforward. For the proof of (5.1, part iii)), let $x=\frac{c+\mu}{\sqrt{2}} \sqrt{n}$ and let $R(x)=\left(x \sqrt{\frac{2}{n}}-c-\varepsilon\right)^{p}(1-\Phi(x))$. Then $x \geq \frac{c+\varepsilon}{\sqrt{2}} \sqrt{n}$ and

$$
\frac{d}{d x} \log R(x)=\frac{p \sqrt{\frac{2}{n}}}{x \sqrt{\frac{2}{n}}-c-\varepsilon}-\frac{\varphi(x)}{1-\Phi(x)} .
$$

Further,

$$
\frac{d^{2}}{d x^{2}} \log R(x)=\frac{-p \frac{2}{n}}{\left(x \sqrt{\frac{2}{n}}-c-\varepsilon\right)^{2}}-\varphi(x) \frac{\varphi(x)-x(1-\Phi(x))}{1-\Phi(x)}<0
$$

because (see e.g. Feller (1968, p. 175))

$$
\varphi(x)-x(1-\Phi(x))>0 \text { for } x \geq 0 \text {. }
$$


So, $\frac{d}{d x} \log R(x)$ is strictly decreasing in $x$ with $\frac{d}{d x} \log R(x) \rightarrow \infty$ as $x \rightarrow \sqrt{\frac{n}{2}}(c+\varepsilon)$. Further, $\frac{d}{d x} \log R(x) \rightarrow-\infty$ as $x \rightarrow \infty$ because, by $(5.3), \varphi(x) /(1-\Phi(x))>x$ for $x \geq 0$. This shows that $\frac{d}{d x} \log R(x)$ has exactly one zero on $\left(\sqrt{\frac{n}{2}}(c+\varepsilon), \infty\right)$, which proves the result.

In the following lemma, the behaviour of $\mu_{m}(c, \varepsilon)$ and of $R\left(\mu_{m}(c, \varepsilon), \delta_{c}, \varepsilon\right)$ as a function of $c$ is studied.

\section{Lem ma 5.2.}

i) For each $\varepsilon, \mu_{m}(c, \varepsilon)$ is continuous and strictly decreasing in $c$,

ii) for each $\varepsilon, \mu_{m}(c, \varepsilon)$ converges to $\varepsilon$ as $c \rightarrow \infty$,

iii) for each $\varepsilon, R\left(\mu_{m}(c, \varepsilon), \delta_{c}, \varepsilon\right)$ is continuous and strictly decreasing in $c$ and converges to 0 as $c \rightarrow \infty$,

iv) $R\left(\mu_{m}(c, \varepsilon), \delta_{c}, \varepsilon\right)$ is, for each $c>0$, strictly decreasing in $\varepsilon$.

Proof. By Theorem 3.1 and Lemma 5.1, part iii), $\mu_{m}(c, \varepsilon)$ is the unique solution, in $\mu$, to $\frac{d}{d \mu}(\mu-\varepsilon)^{p}\left(1-\Phi\left(\frac{c+\mu}{\sqrt{2}} \sqrt{n}\right)\right)=0, \mu>\varepsilon$, or equivalently, to

$$
\left\{\begin{array}{l}
p \sqrt{\frac{2}{n}}\left(1-\Phi\left(\frac{c+\mu}{\sqrt{2}} \sqrt{n}\right)\right)-(\mu-\varepsilon) \varphi\left(\frac{c+\mu}{\sqrt{2}} \sqrt{n}\right)=0 \\
\mu>\varepsilon
\end{array}\right.
$$

This shows that $\mu_{m}(c, \varepsilon)$ has a derivative with respect to $c$ for all $\varepsilon \geq 0$ and all $c>0$. Further, ii) follows from (5.4) and the fact that $\lim _{y \rightarrow \infty} \frac{1-\Phi(y)}{\varphi(y)}=0$.

For the second part of $i$ ), it is sufficient to note that (see (5.2))

$$
\frac{d}{d \mu} \log R\left(\mu, \delta_{c}, \varepsilon\right)=\frac{p \sqrt{\frac{2}{n}}}{\mu-\varepsilon}-\frac{\varphi\left(\frac{c+\mu}{\sqrt{2}} \sqrt{n}\right)}{1-\Phi\left(\frac{c+\mu}{\sqrt{2}} \sqrt{n}\right)}
$$

is strictly decreasing in $c$ because (by $(5.3)) \varphi(x) /(1-\Phi(x))$ is strictly increasing in $x$ for $x \geq 0$. 
For the proof of iii) note that the first part follows from Theorem 3.1, Lemma 5.1, part iii) and the first part of i) of the present lemma. The second part of iii) follows from the fact that, by Lemma 5.1 , part ii), $R\left(\mu, \delta_{c}, \varepsilon\right)$ is strictly decreasing in $c$ for each $\mu>\varepsilon$. For the third part of iii) note that, by Theorem 3.1 and Lemma 5.1, part iii), $\max _{\mu \geq 0} R\left(\mu, \delta_{c}, \varepsilon\right)=\left(\mu_{m}(c, \varepsilon)-\varepsilon\right)^{p} \Phi\left(\frac{-c-\mu_{m}(c, \varepsilon)}{\sqrt{2}} \sqrt{n}\right)$. The result then follows from the fact that, by part ii) of the present lemma, $\mu_{m}(c, \varepsilon)$ converges to $\varepsilon$ as $c \rightarrow \infty$.

For the proof of iv) note that, for every fixed $\mu$ and every $c>0, R\left(\mu, \delta_{c}, \varepsilon\right)$ is strictly decreasing in $\varepsilon$ for $\varepsilon<\mu$. The result then follows from the fact that, by Lemma 5.1, part iii), $\max _{\mu \geq 0} R\left(\mu, \delta_{c}, \varepsilon\right)$ is attained for a value of $\mu>\varepsilon$.

Finally, Lemma 5.3 below gives the behaviour of $\mathcal{E}_{\theta} S_{c}$ as a function of $c$ and of $\mu$.

\section{Lem ma 5.3.}

i) For each $\mu, P_{\theta}\left(S_{c}=1\right)=1$ if $c=0$,

ii) for each $\mu, \mathcal{E}_{\theta} S_{c}$ is strictly increasing in $c$ and converges to 2 as $c \rightarrow \infty$,

iii) for each $c>0, \mathcal{E}_{\theta} S_{c}$ is strictly decreasing in $\mu$ and converges to 1 as $\mu \rightarrow \infty$.

Proof. Part i) is trivially true. Further, part ii) follows immediately from Theorem 3.2. For part iii) note that, for all $\mu, c>0, \sqrt{\frac{2}{n}} \frac{d}{d \mu} \mathcal{E}_{\theta} S_{c}=-\varphi\left(\frac{c-\mu}{\sqrt{2}} \sqrt{n}\right)+$ $\varphi\left(\frac{c+\mu}{\sqrt{2}} \sqrt{n}\right)<0$ and that, for all $c \geq 0, \lim _{\mu \rightarrow \infty}\left(\Phi\left(\frac{c-\mu}{\sqrt{2}} \sqrt{n}\right)+\Phi\left(\frac{c+\mu}{\sqrt{2}} \sqrt{n}\right)\right)=$ 1 .

\section{TABLES}

In this section, tables of the values of $\mu_{m}(c, \varepsilon)$, and of $\mathrm{a}\left(R_{0}, c, \varepsilon\right)$ and $\mathrm{b}\left(R_{0}, c, \varepsilon\right)$ for several values of $\mu, c$ and $R_{0}$ are given for the case where $p=2, \varepsilon=0$ and $n=1$. Values of these quantities for the case where $n>1$ or $\varepsilon>0$ can be obtained by using the relations given at the end of Section 3 . 
TABLE 6.1. $\mu_{m}(c, 0)$ for some values of $c$ and $n=1$

\begin{tabular}{|l|c||l|c||l|l||l|c|}
\hline$c$ & $\mu_{m}(c, 0)$ & $c$ & $\mu_{m}(c, 0)$ & $c$ & $\mu_{m}(c, 0)$ & $\mu_{m}(c, 0)$ & $c$ \\
\hline \hline .0 & 1.684 & .8 & 1.417 & 1.6 & 1.201 & 2.4 & 1.027 \\
.05 & 1.665 & .85 & 1.402 & 1.65 & 1.189 & 2.45 & 1.018 \\
.1 & 1.647 & .9 & 1.387 & 1.7 & 1.177 & 2.5 & 1.008 \\
.15 & 1.630 & .95 & 1.372 & 1.75 & 1.165 & 2.55 & .999 \\
.2 & 1.612 & 1.0 & 1.358 & 1.8 & 1.154 & 2.6 & .990 \\
.25 & 1.594 & 1.05 & 1.344 & 1.85 & 1.142 & 2.65 & .981 \\
.3 & 1.577 & 1.1 & 1.330 & 1.9 & 1.131 & 2.7 & .972 \\
.35 & 1.560 & 1.15 & 1.316 & 1.95 & 1.120 & 2.75 & .963 \\
.4 & 1.544 & 1.2 & 1.303 & 2.0 & 1.109 & 2.8 & .954 \\
.45 & 1.527 & 1.25 & 1.289 & 2.05 & 1.098 & 2.85 & .946 \\
.5 & 1.511 & 1.3 & 1.276 & 2.1 & 1.088 & 2.9 & .937 \\
.55 & 1.494 & 1.35 & 1.263 & 2.15 & 1.077 & 2.95 & .929 \\
.6 & 1.479 & 1.4 & 1.250 & 2.2 & 1.067 & 3.0 & .921 \\
.65 & 1.463 & 1.45 & 1.238 & 2.25 & 1.057 & & \\
.7 & 1.447 & 1.5 & 1.225 & 2.3 & 1.047 & & \\
.75 & 1.432 & 1.55 & 1.213 & 2.35 & 1.037 & & \\
\hline
\end{tabular}


TABLE 6.2. $\mathbf{a}\left(R_{0}, c, 0\right)$ and $\mathbf{b}\left(R_{0}, c, 0\right)$ for some values of $\left(c, R_{0}\right)$ and $n=1$

\begin{tabular}{|c|c|c||c|c||c|c||c|c||c|c||c|c|}
\hline$R_{0}$ & $a(.1)$ & $b(.1)$ & $a(.2)$ & $b(.2)$ & $a(.3)$ & $b(.3)$ & $a(.4)$ & $b(.4)$ & $a(.5)$ & $b(.5)$ & $a(.6)$ & $b(.6)$ \\
\hline \hline .01 & .153 & 4.571 & .158 & 4.450 & .164 & 4.327 & .171 & 4.204 & .178 & 4.080 & .186 & 3.955 \\
.02 & .221 & 4.222 & .229 & 4.096 & .238 & 3.969 & .249 & 3.841 & .260 & 3.711 & .273 & 3.580 \\
.03 & .275 & 3.999 & .287 & 3.870 & .299 & 3.739 & .313 & 3.606 & .328 & 3.472 & .345 & 3.335 \\
.04 & .323 & 3.830 & .337 & 3.698 & .352 & 3.563 & .369 & 3.427 & .389 & 3.288 & .410 & 3.146 \\
.05 & .367 & 3.692 & .384 & 3.556 & .402 & 3.418 & .422 & 3.278 & .446 & 3.134 & .472 & 2.987 \\
.06 & .408 & 3.573 & .427 & 3.434 & .448 & 3.293 & .473 & 3.148 & .500 & 3.000 & .532 & 2.846 \\
.07 & .448 & 3.468 & .469 & 3.326 & .494 & 3.181 & .522 & 3.032 & 554 & 2.879 & .592 & 2.719 \\
.08 & .485 & 3.373 & .510 & 3.228 & .538 & 3.079 & .570 & 2.926 & .607 & 2.766 & .652 & 2.599 \\
.09 & .522 & 3.285 & .549 & 3.137 & .581 & 2.984 & .618 & 2.826 & .661 & 2.661 & .714 & 2.485 \\
.10 & .558 & 3.204 & .589 & 3.052 & .624 & 2.895 & .666 & 2.732 & .716 & 2.559 & .779 & 2.374 \\
.15 & .735 & 2.851 & .786 & 2.678 & .848 & 2.493 & .930 & 2.289 & 1.045 & 2.050 & 1.269 & 1.702 \\
.20 & .923 & 2.539 & 1.010 & 2.329 & 1.136 & 2.079 & 1.428 & 1.663 & & & & \\
.25 & 1.155 & 2.210 & 1.364 & 1.877 & & & & & & & & \\
\hline
\end{tabular}

\begin{tabular}{|c|c|c||c|c||r|r||r|r||l|l||l|l|}
\hline$R_{0}$ & $a(.7)$ & $b(.7)$ & $a(.8)$ & $b(.8)$ & $a(.9)$ & $b(.9)$ & $a(1.0)$ & $b(1.0)$ & $a(1.5)$ & $b(1.5)$ & $a(2.0)$ & $b(2.0)$ \\
\hline \hline .01 & .195 & 3.829 & .205 & 3.701 & .216 & 3.572 & .228 & 3.441 & .317 & 2.752 & .515 & 1.944 \\
.02 & .287 & 3.447 & .303 & 3.311 & .321 & 3.174 & .342 & 3.033 & .506 & 2.261 & & \\
.03 & .364 & 3.196 & .387 & 3.053 & .412 & 2.907 & .441 & 2.757 & .715 & 1.871 & & \\
.04 & .435 & 3.000 & .464 & 2.850 & .498 & 2.695 & .537 & 2.534 & 1.108 & 1.348 & & \\
.05 & .503 & 2.834 & .540 & 2.677 & .583 & 2.511 & .636 & 2.336 & & & & \\
.06 & .570 & 2.687 & .615 & 2.520 & .671 & 2.342 & .742 & 2.148 & & & & \\
.07 & .638 & 2.551 & .694 & 2.373 & .765 & 2.178 & .864 & 1.956 & & & & \\
.08 & .708 & 2.422 & .778 & 2.229 & .873 & 2.011 & .1 .026 & 1.734 & & & & \\
.09 & .781 & 2.296 & .871 & 2.083 & 1.007 & 1.823 & & & & & & \\
.10 & .861 & 2.168 & .981 & 1.926 & 1.235 & 1.548 & & & & & & \\
\hline
\end{tabular}




\section{ACKNOWLEDGEMENT}

Thanks are due to Professor Willem R. van Zwet, University of Leiden, for suggesting the possible use of loss functions as a generalization for the use of $\varepsilon$-best populations.

\section{BIBLIOGRAPHY}

Bechhofer, R.E. (1954). "A single-sample multiple decision procedure for ranking means of normal populations with known variances", Ann. Math. Statist., 25, 16-39.

Bickel, P.J. and Yahav, J.A. (1977). "On selecting a set of good populations", Statistical Decision Theory and Related Topics, II (S.S. Gupta and D.S. Moore, Eds.), Academic Press, New York, 37-55.

Deely, J.J. and Gupta, S.S. (1968). "On the properties of subset selection procedures", Sankhyā Ser. A, 30, 37-50.

Desu, M.M. (1970). “A selection problem”, Ann. Math. Statist., 41, 1596-1603.

Desu, M.M. and Bristol, D.R. (1985). "Equivalence or selection: a distribution-free approach", Biometrical J., 27, 491-500.

Fabian, V. (1962). "On multiple decision methods for ranking population means", Ann. Math. Statist., 33, 248-254.

Feller, W. (1968). An Introduction to Probability Theory and its Applications, Vol. 1 (3rd ed.), Wiley, New York.

Gill, A.N. and Sharma, S.K. (1993). "A selection procedure for selecting good exponential populations", Biometrical J., 35, 361-369.

Gupta, S.S. (1956). "On a decision rule for a problem in ranking means", Ph.D.thesis, Department of Statistics, University of North Carolina, Chapel Hill, N.C.

Gupta, S.S. (1965). "On some multiple decision (selection and ranking) rules", Technometrics, 7, 225-245.

Gupta, S.S. and Kim, W-C. (1981). "On the problem of selecting good populations", Comm. Statist. Theory Methods A, 10, 1043 - 1077.

Gupta, S.S. and Panchapakesan, S. (1979). Multiple Decision Procedures: Theory and Methodology of Selecting and Ranking Populations, John Wiley \& Sons, New York, Toronto. 
Gupta S.S. and Panchapakesan, S. (1985). "Subset selection procedures: review and assessment", Amer. J. Math. Management Sci., Vol 5,

Nos. 3 \& 4, 235-311.

Hsu, J.C. (1984). "Constrained simultaneous confidence intervals for multiple comparisons with the best", Ann. Statist., 12, 1136-1144.

van der Laan, P. (1992). "Subset selection of an almost best treatment", Biometrical J., 34, 647-656.

Lam, K. and Yu, P.L.H. (1994). "A predictive approach for the selection of a fixed number of good treatments", Comm. Statist. Theory Methods, 23, 2469-2492.

Lehmann, E.L. (1963). "A class of selection procedures based on ranks", Math. Ann., 150, 268-275.

Panchapakesan, S. and Santner, T.J. (1977). "Subset selection procedures for $\Delta_{p^{-}}$ superior populations", Comm. Statist. Theory Methods A, 6, 1081-1090.

Studden, W.J. (1967). "On selecting a subset of $k$ populations containing the best", Ann. Math. Statist., 38, 1072-1078. 